\title{
IMPRIMITIVE GAUSSIAN SUMS AND THETA FUNCTIONS OVER A NUMBER FIELD
}

\author{
JACOB NEMCHENOK
}

\begin{abstract}
We obtain a reduction formula for an imprimitive Gaussian sum with a numerical character in an algebraic number field, i.e. a formula that expresses that sum as a product of several elementary factors times a primitive, proper, normed Gaussian sum (formulae (16) and (19)). We also introduce Gaussian sums with Hecke characters and derive a similar reduction formula for them. The derivation is based on an inversion formula for a multivariable theta function associated with the number field, twisted with the numerical character.
\end{abstract}

\section{INTRODUCTION}

Let $K$ be an algebraic number field with the ring of integers $o$ and the absolute different $\mathfrak{d}, \mathfrak{m}$ an integral ideal of $K, \chi$ a numerical character $\bmod \mathfrak{m}, n \in$ $\mathfrak{m}^{-1} \mathfrak{d}-1$. The Gaussian sums over $K$

$$
\tau_{n}(\chi)=\sum_{\substack{a \in \mathfrak{o} \\ \bmod \mathrm{m}}} \chi(a) e^{2 \pi i \operatorname{Tr}_{K / \mathbb{Q}}(n a)}
$$

introduced by Hecke in [3 and 4], generalize the classical Gaussian sums with Dirichlet characters $(K=\mathbb{Q})$. Assume that $\chi$ is primitive and $\tau_{n}(\chi) \neq 0$ (i.e. $\tau_{n}(\chi)$ is proper primitive-see $\left.\S 5\right)$. By the well-known product formula for Gaussian sums [8, Propositions 6.5 and 6.7], $\tau_{n}(\chi)$ can be written as a product of proper primitive Gaussian sums $\tau_{m}(\psi)$, where $\psi$ is a character $\bmod \mathfrak{p}^{e}, \mathfrak{p}$ a prime ideal. The latter can be explicitly evaluated if $e>1$ (see [9] for the case $K=\mathbb{Q},[6]$ for the general case); for $e=1$, we get Gaussian sums over the finite fields $\mathfrak{o} / \mathfrak{p}$. Also, $\tau_{m}(\psi)$ coincide, up to a root of unity, with $\mathfrak{p}$-adic Gaussian sums [8, Corollary 1, Proposition 6.8]. Thus $\tau_{n}(\chi)$ can be explicitly evaluated or expressed in terms of more well-known Gaussian sums.

In this paper we consider a complementary question: given an arbitrary Gaussian sum $\tau_{n}(\chi)$, express it in terms of a proper primitive Gaussian sum. More precisely, we obtain an elementary expression (which we call a reduction formula) for the quotient $\tau_{n}(\chi) / \tau(\psi)$, where $\psi$ is the primitive character associated to $\chi$ and $\tau(\psi)$ is a normed Gaussian sum for $\psi$ (see $\S 6$ ). In the case $K=\mathbb{Q}$, this quotient was computed by Hasse $[1, \S 20.1$.iv], by a series of

Received by the editors August 1, 1988 and, in revised form, March 6, 1990.

1980 Mathematics Subject Classification (1985 Revision). Primary 11F27, 11L05; Secondary $11 \mathrm{~F} 37,11 \mathrm{~F} 41$. 
elementary reduction steps. He later generalized the formula (and its proof) to Gaussian sums for ray class characters over a number field [2, $\S 4.2]$.

Our proof of the reduction formula for Gaussian sums with numerical characters, given in $\S 10$, is entirely different and is based on the transformation properties of multidimensional theta functions with numerical characters. It was inspired by the paper of Joris [5], who showed that the reduction formula in the case $K=\mathbb{Q}$ can be derived from the functional equation of the corresponding $L$-function. For arbitrary $K$, however, $L$-functions are unsuitable as they are formed using ideal and not numerical characters. That is why we use theta functions instead.

The connection between theta functions $\vartheta_{\chi}\left(z,\left(\begin{array}{l}u \\ v\end{array}\right), \mathfrak{a}\right)$ and $\vartheta_{\chi}^{(\mu)}\left(z,\left(\begin{array}{l}u \\ v\end{array}\right), \mathfrak{a}\right)$, defined in $\S \S 7$ and 9 , and Gaussian sums $\tau_{n}(\chi)$ is based on the fact that the latter appear in inversion formulae for the former (formulae (14), $\S 8$ and (14a), $\S 9$ ), which hold for primitive $\chi$. In [3], Hecke deduced from these inversion formulae (with $z=(0, y)$ ) the functional equation for the $L$-function $L(s, X)$, where $X$ is a primitive Hecke character inducing $\chi$ (in the sense of Proposition 1); the Gaussian sum $\tau_{n}(\chi)$ appears in that functional equation as well. The theta functions with both $x$ and $y$ variables were introduced by Hecke in [4]. He used their transformation properties to obtain a reciprocity formula for $\tau_{n}(\chi)$, where $\chi$ is primitive quadratic, and as a consequence the quadratic reciprocity law over an arbitrary number field. The $x$ variable gives theta functions the extra property of periodicity which, together with inversion formulae, will play an essential role in the proof of our main result. In fact, these theta functions provide an important example of modular forms of uniform weight $1 / 2$ or of weight $(1 / 2, \ldots, 1 / 2,3 / 2,1 / 2, \ldots, 1 / 2)$ for a congruence subgroup of the Hilbert modular group $S L_{2}(\mathfrak{o})$. The modular properties of these functions are analyzed in detail in [10].

The Hecke characters and corresponding Gaussian sums are introduced for two reasons. First, they are used to define normed Gaussian sums $\tau(\psi)$ (although the definition involves an arbitrary choice); secondly, Hecke characters are general enough so that each numerical character is induced by a Hecke character. This relation between numerical and Hecke characters carries over to corresponding Gaussian sums, and enables us to easily generalize the reduction formula to Hecke characters and in particular to recover Hasse's reduction formula for Gaussian sums with ray class characters (Corollary 2, §10).

I am grateful to Professor H. Stark for pointing my attention to Joris's paper and for stimulating discussions.

\section{Notation}

Throughout the paper, $K$ is an algebraic number field of degree $N$ over $\mathbb{Q}$, having $r_{1}$ real and $r_{2}$ complex embeddings into $\mathbb{C}$. The ring of integers, the group of units, the different and the absolute value of the discriminant of $K$ are denoted by $\mathfrak{o}, U, \mathfrak{o}$, and $D$, respectively. If $a \in K$, the algebraic conjugates of $a$ are denoted by $a^{(\nu)} \quad(\nu=1, \ldots, N)$, and are ordered so that $a^{(\nu)}$ is real for $1 \leq \nu \leq r_{1}$ and $a^{\left(\nu+r_{2}\right)}=\overline{a^{(\nu)}}$ for $r_{1}+1 \leq \nu \leq r_{1}+r_{2}$. (The horizontal bar always denotes complex conjugation.) For $a \in K$, we also put

$$
e(a)=e^{2 \pi i \operatorname{Tr}(a)}, \quad e_{1}(a)=e^{\pi i \operatorname{Tr}(a)},
$$

where $\operatorname{Tr}$ denotes the algebraic trace from $K$ to $\mathbb{Q}$ and $i=\sqrt{-1}$. 
A fractional ideal $\mathfrak{a}$ can be uniquely written as a ratio $\mathfrak{b} / \mathfrak{c}$ of two integral coprime ideals, called the numerator and the denominator of $\mathfrak{a}$, respectively. If $\mathfrak{m}$ is an integral ideal of $K$, we say that $\mathfrak{a}$ is prime to $\mathfrak{m}$ if $\mathfrak{b}$ and $\mathfrak{c}$ are both prime to $\mathfrak{m}$. The greatest common divisor of two integral ideals $\mathfrak{a}$ and $\mathfrak{b}$ is denoted by $(\mathfrak{a}, \mathfrak{b})$. The absolute norm of a fractional ideal $\mathfrak{a}$ is denoted by $N(\mathfrak{a})$. The Möbius function and the Euler phi-function of an integral ideal $\mathfrak{a}$ are denoted by $\mu(\mathfrak{a})$ of $\phi(\mathfrak{a})$, respectively. If $\mathfrak{m}$ is an integral ideal of $K$, we let $I(\mathfrak{m})$ be the group of fractional ideals of $K$ which are prime to $\mathfrak{m}$, and we also put

$$
\begin{gathered}
K(\mathfrak{m})=\left\{a \in K^{*} \mid(a) \in I(\mathfrak{m})\right\}, \\
K_{1}(\mathfrak{m})=\left\{a \in K^{*} \mid \mathfrak{m} \text { divides the numerator of }(a-1)\right\}, \\
P(\mathfrak{m})=\{(a) \mid a \in K(\mathfrak{m})\}, \quad P_{1}(\mathfrak{m})=\left\{(a) \mid a \in K_{1}(\mathfrak{m})\right\} .
\end{gathered}
$$

Our notation for theta functions essentially follows that of [10].

\section{Numerical characters aNd Hecke Characters}

Let $\mathfrak{m}$ be an integral ideal of $K$. Every additive character of the abelian group $\mathfrak{o} / \mathfrak{m}$ of residue classes modulo $\mathfrak{m}$ has the form

$$
e_{n}(a+\mathfrak{m})=e(n a) \quad(a \in \mathfrak{o}) \quad \text { for some } n \in \mathfrak{m}^{-1} \mathfrak{d}^{-1} .
$$

Following Lamprecht, we call $e_{n}$ proper (echte in German) if, as $t$ runs over the residue classes of $\mathfrak{o} \bmod \mathfrak{m}, e_{n t}$ runs over all additive characters of $\mathfrak{o} / \mathfrak{m}$. It is easy to check that the character $e_{n}$ is proper if and only if the ideal $\mathfrak{m o}(n)$ is prime to $\mathfrak{m}$.

A character $\chi$ of the abelian group $(\mathfrak{o} / \mathfrak{m})^{\times}$of reduced residue classes modulo $\mathfrak{m}$ is called a numerical character (or simply a character) $\bmod \mathfrak{m}$. We will think of $\chi$ as a complex-valued function on $\mathfrak{o}$ satisfying, for $a, b \in \mathfrak{o}$,

(i) $\chi(a b)=\chi(a) \chi(b)$,

(ii) $\chi(a)=\chi(b)$ if $a \equiv b(\bmod \mathfrak{m})$,

(iii) $\chi(a)=0$ if $a \notin K(\mathfrak{m})$.

We will also need to evaluate $\chi$ at nonintegral elements of $K$. Namely, if an element $a \in K$ can be written as a ratio $b / c$, where $b, c \in \mathfrak{o}$ and $c \in K(\mathfrak{m})$ (equivalently, if the denominator of $(a)$ is in $I(\mathfrak{m}))$, then we put $\chi(a)=\chi(b) \chi(c)^{-1}$. Thus $\chi$ is defined on $K(\mathfrak{m})$ and $\chi=1$ on $K_{1}(\mathfrak{m})$.

The conjugate character $\bar{\chi}$ is defined by $\bar{\chi}(a)=\overline{\chi(a)}$ whenever $\chi(a)$ makes sense.

Let $\mathfrak{m}$ be an integral ideal of $K$. A Hecke character mod $\mathfrak{m}$ is a homomorphism $X$ of $I(\mathfrak{m})$ into the group $\mathbb{C}^{1}=\{z \in \mathbb{C}|| z \mid=1\}$ such that

$$
X((a))=\lambda(a) \quad\left(a \in K_{1}(\mathfrak{m})\right),
$$

where $\lambda$ is a homomorphism of $K^{*}$ into $\mathbb{C}^{1}$ of the form

$$
\lambda(a)=\prod_{\nu=1}^{r_{1}+r_{2}}\left(\frac{a^{(\nu)}}{\left|a^{(\nu)}\right|}\right)^{l_{\nu}}\left|a^{(\nu)}\right|^{i t_{\nu}}
$$

for some $l_{\nu} \in \mathbb{Z}, t_{\nu} \in \mathbb{R},\left(\nu=1, \ldots, r_{1}+r_{2}\right)$. (This definition is very close to Hecke's original one.) More generally, one can include infinite real primes in the modulus of $X$, but for our purposes this is unnecessary. If $\lambda \equiv 1, X$ is called a ray class character mod $\mathrm{m}$. We call $\lambda$ the infinite part of $X$. 
As usual, we extend $X$ to the semigroup of all integral ideals of $K$ by putting $X(\mathfrak{a})=0$ if $\mathfrak{a} \notin I(\mathfrak{m})$. The conjugate character $\bar{X}$ is defined by $\bar{X}(\mathfrak{a})=\overline{X(\mathfrak{a})}$.

The following proposition (essentially contained in [3]) shows the relation between Hecke characters and numerical characters.

Proposition 1. Let $X$ be a Hecke character mod $\mathfrak{m}$ with infinite part $\lambda$. Then there exists a numerical character $\chi \bmod \mathfrak{m}$ such that

$$
X((a))=\chi(a) \lambda(a) \quad(a \in K(\mathfrak{m})) .
$$

Conversely, given a numerical character $\chi \bmod \mathfrak{m}$, there exists a (nonunique) Hecke character $X$ mod $\mathfrak{m}$ with infinite part $\lambda$ such that (2) holds.

Proof. The first assertion follows directly from the definitions. To prove the second one, we first find $\lambda$ so that $\chi(\varepsilon) \lambda(\varepsilon)=1$ for every $\varepsilon \in U$ (the existence of such $\lambda$ is guaranteed by Dirichlet's unit theorem). Now define $X$ on the group $P(\mathfrak{m})$ by formula (2) (this is unambiguous by the choice of $\lambda$ ), and then extend $X$ to a character (denoted by the same letter) on the group $I(\mathfrak{m})$. Since $\chi=1$ on $K_{1}(\mathfrak{m})$, we get $X=\lambda$ on $K_{1}(\mathfrak{m})$, i.e. $X$ is a Hecke character; and (2) holds by the construction $X$. Q.E.D.

If $X, \chi$ are as in (2), we say that $X$ induces $\chi$.

\section{Primitive AND imprimitive ChaRACTERS}

Recall that a numerical character $\chi \bmod \mathfrak{m}$ is called imprimitive if it is induced by a character of a smaller modulus, i.e. if there exists an ideal $\mathfrak{m}^{\prime}$ strictly dividing $m$ and a numerical character $\chi^{\prime} \bmod \mathfrak{m}^{\prime}$ such that $\chi=\chi^{\prime}$ on $K(\mathfrak{m})$. Otherwise, $\chi$ is called primitive. The smallest ideal $\mathfrak{m}^{\prime}$ as above is called the conductor of $\chi$ and is denoted by $\mathfrak{f}_{\chi}$ (thus $\chi$ is primitive if and only if $\mathfrak{f}_{\chi}=\mathfrak{m}$ ). The character $\chi$ is induced by a unique primitive character $\psi \bmod \mathfrak{f}_{\chi}$, which we call the primitive character associated with $\chi$. The two characters are related by the formula

$$
\chi=\psi \chi_{1},
$$

where $\chi_{1}$ is the principal character mod $\mathfrak{q}, \mathfrak{q}$ being the product of prime ideals dividing $\mathfrak{m}$ but not $\mathfrak{f}_{\chi}$. The definitions for Hecke characters are completely analogous.

Let $X$ be a Hecke character mod $m$ with infinite part $\lambda$, and let $\chi$ be the numerical character mod $m$ induced by $X$. It is natural to ask whether the primitivity of $X$ is related to the primitivity of $\chi$. The answer is stated in Proposition 3. But first we need an auxiliary result.

Proposition 2. Let $X, \lambda$ be as above, and suppose that $X$ is induced by a Hecke character $X^{\prime} \bmod \mathfrak{m}^{\prime}$, where $\mathfrak{m}^{\prime} \mid \mathfrak{m}$, with infinite part $\lambda^{\prime}$. Then $\lambda^{\prime}=\lambda$.

Proof. From the definitions, we get $\lambda^{\prime}(a)=X^{\prime}((a))=X((a))=\lambda(a)$ for all $a \in K_{1}(\mathfrak{m})$. Put $\rho=\lambda^{\prime} \bar{\lambda}$ (where by definition $\bar{\lambda}(a)=\overline{\lambda(a)}$ ). We can view $\lambda, \lambda^{\prime}$, and hence also $\rho$, as continuous characters of the topological group $G=$ $\mathbb{R}^{* r_{1}} \times \mathbb{C}^{* 2 r_{2}}$, and we have just seen that the kernel of $\rho$ contains $K_{1}(\mathfrak{m})(K$ is embedded into $G$ via its conjugates). But $K_{1}(\mathfrak{m})$ is of finite index in the group $K(\mathfrak{m})$, which is dense in $G$ by the Strong Approximation Theorem. Therefore $\rho=1$. Q.E.D. 
Proposition 3. Let $X, \lambda, \chi$ be as above. Then

(i) $X$ is (im) primitive if and only if $\chi$ is (im)primitive;

(ii) the conductor of $X$ is equal to the conductor of $\chi$;

(iii) if $\Psi, \psi$ are the primitive characters associated to $X, \chi$, respectively, then $\psi$ is the numerical character induced by $\Psi$.

Proof. Suppose that $X$ is imprimitive. Then there exists an ideal $\mathfrak{m}^{\prime}$ strictly dividing $\mathfrak{m}$ and a Hecke character mod $\mathfrak{m}^{\prime}$ such that $X=X^{\prime}$ on $K_{1}(\mathfrak{m})$. By the previous proposition, the infinite part of $X^{\prime}$ is $\lambda$. Let $\chi^{\prime}$ denote the numerical character mod $\mathfrak{m}^{\prime}$ induced by $X^{\prime}$. Then for every $a \in K(\mathfrak{m})$ we have $\chi(a)=X((a)) \bar{\lambda}(a)=X^{\prime}((a)) \bar{\lambda}(a)=\chi^{\prime}(a)$, therefore $\chi$ is imprimitive.

Conversely, suppose that $\chi$ is induced by a numerical character $\chi^{\prime} \bmod \mathfrak{m}^{\prime}$, where $\mathfrak{m}^{\prime}$ is an ideal strictly dividing $\mathfrak{m}$. Define a character $\Psi$ on $P_{1}\left(\mathfrak{m}^{\prime}\right)$ by $\Psi((a))=\lambda(a)$. This makes sense because for $\varepsilon \in U \cap K_{1}\left(\mathfrak{m}^{\prime}\right), \chi(\varepsilon)=$ $\chi^{\prime}(\varepsilon)=1$ and consequently $\lambda(\varepsilon)=X((\varepsilon))=1$. A similar computation shows that $X=\lambda$ on $I(\mathfrak{m}) \cap P_{1}\left(\mathfrak{m}^{\prime}\right)$. This and the fact that the groups involved are abelian, enables us to extend $\Psi$ to the group $I(\mathfrak{m}) P_{1}\left(\mathfrak{m}^{\prime}\right)$ by $\Psi(\mathfrak{a}(a))=$ $X(\mathfrak{a}) \lambda(a)$. Extending $\Psi$ further to the whole group $I\left(\mathfrak{m}^{\prime}\right)$ in an arbitrary way, we obtain a character which by construction is a Hecke character and agrees with $X$ on $I(\mathfrak{m})$. Therefore $X$ is imprimitive, and (i) is proved (the second implication in (i) is stated in $[4$, p. 264].

Part (ii) is an immediate consequence of (i). Finally, let $\Psi, \psi$ be as in (iii), and let $\psi^{\prime}$ be the numerical character induced by $\Psi$. By parts (i)-(ii), $\psi^{\prime}$ is primitive $\bmod \mathfrak{f}_{\chi}$, hence $\psi^{\prime}=\psi$, which proves (iii).

\section{GAUSSIAN SUMS WITH NUMERICAL CHARACTERS}

Let $\chi$ be a character $\bmod \mathfrak{m}$. The Gaussian sum for $\chi$ with the parameter $n$ is defined by

$$
\tau_{n}(\chi)=\sum_{\substack{a \in \mathfrak{o} \\ \bmod \mathfrak{m}}} \chi(a) e_{n}(a)=\sum_{\substack{a \in \mathfrak{o} \\ \bmod \mathfrak{m}}} \chi(a) e(n a),
$$

where $n \in \mathfrak{m}^{-1} \mathfrak{d}^{-1}$, so that $(n)=\mathfrak{m}^{-1} \mathfrak{d}^{-1} \mathfrak{n}, \mathfrak{n}$ an integral ideal.

A Gaussian sum $\tau_{n}(\chi)$ will be called proper if the additive character $e_{n}$ is proper (this is in a slight conflict with Hasse's terminology in [2]); it will be called primitive if the multiplicative character $\chi$ is primitive. We recall some elementary properties of $\tau_{n}(\chi)$ in the next proposition.

Proposition 4. With the above notation, we have

$$
\begin{gathered}
\overline{\tau_{n}(\chi)}=\chi(-1) \tau_{n}(\bar{\chi}), \\
\tau_{n}(\chi)=\sum_{\substack{a \in \mathfrak{a} \\
\bmod \mathfrak{m a}}} \chi(a) e(n a) \quad(\mathfrak{a} \in I(\mathfrak{m})),
\end{gathered}
$$

$$
\tau_{n t}(\chi)=\bar{\chi}(t) \tau_{n}(\chi) \text { for all } t \in \mathfrak{n}^{-1} \text { such that } \chi(t) \text { is defined. }
$$

In addition, if $\chi$ is primitive, then

$$
\left|\tau_{n}(\chi)\right|= \begin{cases}0, & \text { if }(\mathfrak{n}, \mathfrak{m})=\mathfrak{o}, \\ N(\mathfrak{m})^{1 / 2}, & \text { otherwise }\end{cases}
$$


(in other words, a primitive Gaussian sum does not vanish if and only if it is proper).

Proof. The formula (4) is obvious. To prove (5), observe that if $\mathfrak{b}=\mathfrak{a} \cap \mathfrak{o}$ ( $=$ the numerator of $\mathfrak{a})$, then a complete system of coset representatives for $\mathfrak{b} \bmod \mathfrak{m} \mathfrak{b}$ is also a complete system of coset representatives for both $\mathfrak{a}$ mod $\mathfrak{m a}$ and $\mathfrak{o} \bmod \mathfrak{m}$. The proof of (6) splits into two cases: (i) $t \in K(\mathfrak{m})$ and (ii) $\chi(t)=0$. In case (i), (6) follows from (5) by putting $\mathfrak{a}=(t)$ and making a substitution $a=b t$. In case (ii), $\bar{\chi}(t)=0$ and by (7), $\tau_{n t}(\chi)=0$, so both sides are zero. As for (7), we may assume, in view of the product formula mentioned in the introduction, that $\mathfrak{m}$ is a prime power. For the elementary proof in that case, see [6, Regel 1, p. 155 and Satz 6, p. 164].

\section{Gaussian sums with Hecke characters. Normed gaUssian sums}

Let $X$ be a Hecke character mod $\mathfrak{m}$ with infinite part $\lambda$, and let $\mathfrak{a}$ be a fractional ideal of $K$ and $n$ a number in $\mathfrak{m}^{-1} \mathfrak{a}^{-1} \mathfrak{d}^{-1}$. We define the Gaussian sum for $X$ with the parameter $n$ and the auxiliary ideal a by

$$
\tau_{n}(X \mid \mathfrak{a})=\sum_{\substack{a \in \mathfrak{a} \\ \text { mod } \mathbf{m} \mathfrak{a} \\(a)=\mathbf{a r}}} X(\mathfrak{r}) \bar{\lambda}(a) e(n a) .
$$

The sum is independent of the choice of coset representatives for $\mathfrak{a}$ mod $\mathfrak{m a}$, thanks to formula (2) and the restriction on the parameter $n$. For $\lambda=1$ this coincides with Hasse's definition of a Gaussian sum for a ray class character in [2].

Every Gaussian sum $\tau_{n}(X \mid \mathfrak{a})$ as above can be reduced to a Gaussian sum for a numerical character. Indeed, if $\mathfrak{c}$ is an integral ideal prime to $m$ such that the ideal $\mathfrak{a} \mathfrak{c}=(c)$ is principal, an easy calculation as in $[2, \S 2]$ shows that

$$
\tau_{n}(X \mid \mathfrak{a})=X(\mathfrak{c}) \bar{\lambda}(c) \tau_{n c}(X \mid \mathfrak{o}),
$$

and equation (2) implies that

$$
\tau_{n}(X \mid \mathfrak{o})=\tau_{n}(\chi),
$$

where $\chi$ is the numerical character mod $m$ induced by $X$. Therefore

$$
\tau_{n}(X \mid \mathfrak{a})=X(\mathfrak{c}) \bar{\lambda}(c) \tau_{n c}(\chi) .
$$

Observe that, unlike the traditional Gaussian sums, $\tau_{n}(X \mid \mathfrak{a})$ generally takes transcendental values. Note also that $\tau_{n}(X \mid \mathfrak{a}) \neq 0$ if and only if $\tau_{n c}(\chi) \neq 0$. By (7), this is equivalent to $(\mathfrak{m a d}(n), \mathfrak{m})=\mathfrak{o}$. Parameters $n, \mathfrak{a}$ satisfying this last condition are called proper. We call the Gaussian sum $\tau_{n}(X \mid \mathfrak{a})$ proper if $n, \mathfrak{a}$ are proper, and primitive if $X$ is primitive.

A proper primitive Gaussian sum with a Hecke character $X$ can be normalized so that it depends only on $X$ and not on the choice of parameters $n, \mathfrak{a}$. Namely, if $X$ is primitive mod $m$ and has infinite part $\lambda$, we define the normed Gaussian sum for $X$ by

$$
\tau(X)=X(\mathfrak{n}) \bar{\lambda}(n) \tau_{n}(X \mid \mathfrak{a}),
$$

where $\tau_{n}(X \mid \mathfrak{a})$ is any proper Gaussian sum for $X$ and $\mathfrak{n}=\operatorname{mad}(n)$. That this is independent on the choice of parameters can be easily checked using (8) and 
(6). Even better, by definition

$$
\tau(X)=\sum_{\substack{a \in \mathfrak{a} \\ \mathfrak{m} \mathfrak{m} \mathfrak{m} \mathfrak{a} \\(a)=\mathfrak{a r}}} X(\mathfrak{n r}) \bar{\lambda}(n a) e(n a) .
$$

Making a substitution $b=n a$, we get

$$
\tau(X)=\tau_{1}\left(X \mid \mathfrak{m}^{-1} \mathfrak{d}^{-1}\right),
$$

which visibly depends only on $X$ !

Unfortunately, such a canonical normalization does not exist for proper primitive Gaussian sums with numerical characters. If $\chi$ is a primitive numerical character mod $\mathfrak{m}$, the best we can do is fix a primitive Hecke character $X$ that induces $\chi$ (see Propositions 1 and 3) and define a normed Gaussian sum for $\chi$ by

$$
\tau(\chi)=\tau(X),
$$

or, using the formula above,

$$
\tau(\chi)=\tau_{n}(\chi) X(\mathfrak{n}) \bar{\lambda}(n),
$$

where $\tau_{n}(\chi)$ is any proper Gaussian sum for $\chi, \mathfrak{n}=\mathfrak{m} \mathfrak{d}(n)$, and $\lambda$ is the infinite part of $X$.

\section{TheTA FUNCTIONS OVER $K$}

Let $\mathfrak{H}_{1}=\{x+y i \in \mathbb{C} \mid y>0\}$ be the complex upper half-plane and $\mathfrak{H}_{2}=$ $\{x+y k \in \mathbb{H} \mid x \in \mathbb{C}, y>0\}$ the quaternionic upper half-space, where $\mathbb{H}=$ $\{a+b i+c j+d k \mid a, b, c, d \in \mathbb{R}\}$ is the quaternion algebra over $\mathbb{R}$ and $\mathbb{C}$ is identified with the subset $\{a+b i\}$ of $\mathbb{H}$. The generalized upper half-space over the field $K$, denoted by $\mathfrak{H}_{K}$, is the set of $N$-tuples $z=\left(z^{(\nu)}\right)_{\nu=1, \ldots, N}$ such that

(i) $z^{(\nu)}=x^{(\nu)}+y^{(\nu)} i \in \mathfrak{H}_{1} \quad\left(1 \leq \nu \leq r_{1}\right)$,

(ii) $z^{(\nu)}=x^{(\nu)}+y^{(\nu)} k \in \mathfrak{H}_{2} \quad\left(r_{1}+1 \leq \nu \leq N\right)$,

(iii) $x^{\left(\nu+r_{2}\right)}=\overline{x^{(\nu)}}, y^{\left(\nu+r_{2}\right)}=y^{(\nu)}\left(r_{1}+1 \leq \nu \leq r_{1}+r_{2}\right)$.

We will sometimes write $z=(x, y)$ for $z \in \mathfrak{H}_{K}$, where $x, y$ are as in (i)-(iii).

The group $S L_{2}(K)$ acts on $\mathfrak{H}_{K}$ as follows:

$$
\begin{aligned}
\left(\begin{array}{ll}
a & b \\
c & d
\end{array}\right) \circ z=\left[\left(a^{(\nu)} z^{(\nu)}+b^{(\nu)}\right)\left(c^{(\nu)} z^{(\nu)}+d^{(\nu)}\right)^{-1}\right]_{\nu=1, \ldots, N} \\
\\
\qquad \text { for }\left(\begin{array}{ll}
a & b \\
c & d
\end{array}\right) \in S L_{2}(K), \quad z \in \mathfrak{H}_{K} .
\end{aligned}
$$

The following notation will be used (for $z=(x, y) \in \mathfrak{H}_{K}$ ):

$$
\begin{aligned}
-z^{-1} & =\left(\begin{array}{cc}
0 & -1 \\
1 & 0
\end{array}\right) \circ z=\left(-z^{(\nu)^{-1}}\right)_{\nu=1, \ldots, N}, \\
z+b & =\left(\begin{array}{cc}
1 & b \\
0 & 1
\end{array}\right) \circ z=\left(x^{(\nu)}+b^{(\nu)}, y^{(\nu)}\right)_{\nu=1, \ldots, N} \quad(b \in K), \\
a^{2} z & =\left(\begin{array}{cc}
a & 0 \\
0 & a^{-1}
\end{array}\right) \circ z=\left(a^{(\nu)^{2}} x^{(\nu)},\left|a^{(\nu)}\right|^{2} y^{(\nu)}\right)_{\nu=1, \ldots, N} \quad\left(a \in K^{*}\right) .
\end{aligned}
$$


Let us now define a theta function over $k$ with characteristics $u, v$ by the formula

$$
\begin{array}{r}
\vartheta\left(z,\left(\begin{array}{l}
u \\
v
\end{array}\right), \mathfrak{a}\right)=\sum_{a \in \mathfrak{a}} \exp \left\{\pi i\left[\sum_{\nu=1}^{N} x^{(\nu)}\left(a^{(\nu)}+v^{(\nu)}\right)^{2}-2 u^{(\nu)} a^{(\nu)}-u^{(\nu)} v^{(\nu)}\right]\right. \\
\left.-\pi \sum_{\nu=1}^{N} y^{(\nu)}\left|a^{(\nu)}+v^{(\nu)}\right|^{2}\right\} .
\end{array}
$$

$\left(z=(x, y) \in \mathfrak{H}_{K}, u, v \in K, \mathfrak{a}\right.$ is a fractional ideal of $K$.) This function is taken form [10]. Hecke [4, $\S 57$, formula (185)], defines a function $\theta(t, z, \omega ; \mathfrak{a})=\vartheta\left((2 \omega, t),\left(\begin{array}{l}0 \\ v\end{array}\right), \mathfrak{a}\right)$. For the sake of brevity, we will write

$$
\vartheta\left(z,\left(\begin{array}{l}
u \\
v
\end{array}\right), \mathfrak{a}\right)=\sum_{a \in \mathfrak{a}} e_{1}\left[x\left(a+u_{\mathfrak{b}}\right)^{2}+i y|a+v|^{2}-2 u a-u v\right]
$$

with obvious conventions.

The two basic transformation properties of this function are

(i) Periodicity in the $x$ variable when $v=0$ :

$$
\vartheta\left(z+b,\left(\begin{array}{l}
u \\
0
\end{array}\right), \mathfrak{a}\right)=\vartheta\left(z,\left(\begin{array}{l}
u \\
0
\end{array}\right), \mathfrak{a}\right) \quad\left(b \in(2) \mathfrak{a}^{-2} \mathfrak{d}^{-1}\right),
$$

(ii) the inversion formula

$$
\vartheta\left(-z^{-1},\left(\begin{array}{c}
-v \\
u
\end{array}\right), \mathfrak{a}^{-1} \mathfrak{d}^{-1}\right)=N(\mathfrak{a}) D^{1 / 2} N(-i z)^{1 / 2} \vartheta\left(z,\left(\begin{array}{c}
u \\
v
\end{array}\right), \mathfrak{a}\right) .
$$

Here for $z=(x, y) \in \mathfrak{H}_{K}, N(-i z)^{1 / 2}$ is defined as a product

$$
\prod_{\nu=1}^{r_{1}}\left(-i z^{(\nu)}\right)^{1 / 2} \prod_{\nu=r_{1}+1}^{r_{1}+r_{2}}\left\|z^{(\nu)}\right\|^{1 / 2}
$$

where $\left\|z^{(\nu)}\right\|=\left|x^{(\nu)}\right|^{2}+y^{(\nu)^{2}}$ is the quaternionic norm of $z^{(\nu)} \quad\left(r_{1}+1 \leq \nu \leq\right.$ $\left.r_{1}+r_{2}\right)$; the square root is chosen to be positive when $z^{(\nu)}$ is purely imaginary $\left(1 \leq \nu \leq r_{1}\right)$ and always positive for $r_{1}+1 \leq \nu \leq r_{1}+r_{2}$.

The property (i) is obvious; as for (ii), it is shown in [10] that $\vartheta\left(z,\left(\begin{array}{l}u \\ v\end{array}\right), \mathfrak{a}\right)$ is a specialization of a "twist" of the symplectic theta function

$$
\vartheta^{\alpha}\left(\begin{array}{l}
v \\
u
\end{array}\right)(\Omega)=\sum_{n \in \mathbb{Z}^{N}} \exp \left\{\pi i\left[^{t}(n+v) \Omega(n+v)-2^{t} n u-{ }^{t} v u\right]\right\}
$$

[7, §II.5, formula $\left.\left(5.3^{\prime}\right)\right]$; here $u, v \in \mathbb{C}^{N}, \Omega$ is an $N \times N$ complex symmetric matrix whose imaginary part is positive definite, ${ }^{t} A$ denotes the transpose of a matrix $A$, and all vectors are column vectors. The formula (10) is then easily deduced from the well-known inversion formula for $\vartheta^{\alpha}\left(\begin{array}{c}v \\ u\end{array}\right)(\Omega)[7, \S \amalg I .5$, formula (5.6)]. For the proof of $(10)$ in the case $u=v=0$, see also [4, §57, Theorem 162].

We note for future reference

$$
N\left[-i\left(a^{2} z\right)\right]^{1 / 2}=N((a)) N(-i z)^{1 / 2}, \quad N\left[-i\left(-z^{-1}\right)\right]^{1 / 2}=N(-i z)^{-1 / 2} .
$$


The following "rescaling" formula will also be useful:

$$
\vartheta\left(a^{2} z,\left(\begin{array}{c}
u \\
v
\end{array}\right), \mathfrak{a}\right)=\vartheta\left(z,\left(\begin{array}{c}
u / a \\
a v
\end{array}\right),(a) \mathfrak{a}\right) \quad\left(a \in K^{*}\right) .
$$

\section{THETA FUNCTIONS WITH CHARACTERS}

Let $\chi$ be a character $\bmod \mathfrak{m}$. Define

$$
\vartheta_{\chi}\left(z,\left(\begin{array}{l}
u \\
v
\end{array}\right), \mathfrak{a}\right)=\sum_{a \in \mathfrak{a}} \chi(a) e_{1}\left[x(a+v)^{2}+i y|a+v|^{2}-2 a u-u v\right]
$$

where $z \in H, u, v \in K, \mathfrak{a}$ is a fractional ideal whose denominator is prime to $\mathfrak{m}$, and the notational conventions for $e_{1}[\cdots]$ are as in $\S 7$. Thus, theta functions of the previous section are particular instances of $\vartheta_{\chi}$ when $\chi=$ $\chi_{1}$, the principal character mod $o$. On the other hand, grouping together the elements $a \in \mathfrak{a}$ with the same residue $\bmod \mathfrak{m}$, we see that each $\vartheta_{\chi}$ is a linear combination of $\vartheta$ 's:

$$
\vartheta_{\chi}\left(z,\left(\begin{array}{l}
u \\
v
\end{array}\right), \mathfrak{a}\right)=\sum_{\substack{b \in \mathfrak{a} \\
\bmod \mathfrak{m} \mathfrak{a}}} \chi(b) e_{1}(-u b) \vartheta\left(z,\left(\begin{array}{c}
u \\
v+b
\end{array}\right), \mathfrak{m a}\right) .
$$

Using this formula and the inversion formula (10), we obtain

Proposition 5. Let $\chi$ be a primitive character $\bmod \mathfrak{m}$, and let $(n)=\mathfrak{m}^{-1} \mathfrak{d}^{-1} \mathfrak{n}$, $\mathfrak{n}$ an integral ideal prime to $\mathfrak{m}$. Then the following inversion formula holds:

$$
\begin{aligned}
& \vartheta_{\bar{\chi}}\left(-n^{2} z^{-1},\left(\begin{array}{c}
-n v \\
u / n
\end{array}\right), \mathfrak{n}^{-1} \mathfrak{a}^{-1}\right) \\
& \quad=\chi(-1) \tau_{n}(\bar{\chi}) N(\mathfrak{a}) D^{1 / 2} N(-i z)^{1 / 2} \vartheta_{\chi}\left(z,\left(\begin{array}{c}
u \\
v
\end{array}\right), \mathfrak{a}\right) .
\end{aligned}
$$

Proof. Using successively (13), (12), and (10), we get

$$
\begin{aligned}
\vartheta_{\bar{\chi}}\left(-n^{2} z^{-1},\left(\begin{array}{c}
-n v \\
u / n
\end{array}\right), \mathfrak{n}^{-1} \mathfrak{a}^{-1}\right) & \\
= & \sum_{\substack{b \in \mathfrak{n}^{-1} \mathfrak{a}^{-1} \\
\bmod \mathbf{m} \mathfrak{n}^{-1} \mathfrak{a}^{-1}}} \bar{\chi}(b) e_{1}(n v b) \vartheta\left(-n^{2} z^{-1},\left(\begin{array}{c}
-n v \\
u / n+b
\end{array}\right), \mathfrak{m n}^{-1} \mathfrak{a}^{-1}\right) \\
= & \sum_{\substack{b \in \mathfrak{n}^{-1} \mathfrak{a}^{-1} \\
\bmod m \mathfrak{n}^{-1} \mathfrak{a}^{-1}}} \bar{\chi}(b) e_{1}(n v b) \vartheta\left(-z^{-1},\left(\begin{array}{c}
-v \\
u+n b
\end{array}\right), \mathfrak{a}^{-1} \mathfrak{d}^{-1}\right) \\
= & N(\mathfrak{a}) D^{1 / 2} N(-i z)^{1 / 2} \sum_{\substack{b \in \mathfrak{n}^{-1} \mathfrak{a}^{-1} \\
\bmod \mathfrak{m} \mathfrak{n}^{-1} \mathfrak{a}^{-1}}} \bar{\chi}(b) e_{1}(n v b) \vartheta\left(z,\left(\begin{array}{c}
u+n b \\
v
\end{array}\right), \mathfrak{a}\right) \\
= & N(\mathfrak{a}) D^{1 / 2} N(-i z)^{1 / 2} \sum_{\substack{b \in \mathfrak{n}^{-1} \mathfrak{a}^{-1} \\
\bmod \mathfrak{m} \mathfrak{n}^{-1} \mathfrak{a}^{-1}}} \bar{\chi}(b) \\
& \left.\times \sum_{a \in \mathfrak{a}} e_{1}\left[x(a+v)^{2}+i y|a+v|^{2}-2(u+n b) a-(u+n b) v+n v b\right)\right]
\end{aligned}
$$




$$
\begin{aligned}
& =N(\mathfrak{a}) D^{1 / 2} N(-i z)^{1 / 2} \sum_{a \in \mathfrak{a}}\left[\sum_{\substack{b \in \mathfrak{n}^{-1} \mathfrak{a}^{-1} \\
\bmod \operatorname{mn} \mathfrak{n}^{-1} \mathfrak{a}^{-1}}} \bar{\chi}(b) e(-n a b)\right] \\
& \times e_{1}\left[x(a+v)^{2}+i y|a+v|^{2}-2 u a-u v\right] .
\end{aligned}
$$

Finally, the sum in brackets is equal to $\tau_{-n a}(\bar{\chi})=\chi(-1) \chi(a) \tau_{n}(\bar{\chi})$ by virtue of (5) and (6). Q.E.D.

Theta functions with zero characteristics will be written as $\vartheta_{\chi}(z, \mathfrak{a})$. Observe that $\vartheta_{\chi}(z, \mathfrak{a})$ vanishes identically if and only if $\chi$ is odd.

Let $\psi$ be the primitive character associated with $\chi$, and let the ideal $\mathfrak{q}$ be as in (3). Then $\vartheta_{\chi}\left(z,\left(\begin{array}{l}u \\ 0\end{array}\right), \mathfrak{a}\right)$ is a linear combination of $\vartheta_{\psi}$ 's:

$$
\vartheta_{\chi}\left(z,\left(\begin{array}{c}
u \\
0
\end{array}\right), \mathfrak{a}\right)=\sum_{\mathfrak{b} \mid \mathfrak{q}} \mu(\mathfrak{b}) \vartheta_{\psi}\left(z,\left(\begin{array}{c}
u \\
0
\end{array}\right), \mathfrak{a} \mathfrak{b}\right) .
$$

This follows from (3) and the identity

$$
\chi_{1}(a)=\sum_{\mathfrak{b} \mid((a), \mathfrak{q})} \mu(\mathfrak{b}) \quad(a \in \mathfrak{o})
$$

Remark. Let the notation be as in Proposition 5. Hecke noticed that the equality $\left|\tau_{n}(\chi)\right|=N(\mathfrak{m})^{1 / 2}$ in (7) drops out of the inversion formula for a theta function with $\chi$. Indeed, suppose first that $\chi$ is even. By (14),

$$
\begin{gathered}
\vartheta_{\chi}(z, \mathfrak{o})=\bar{\chi}(-1) \tau_{n}(\chi) D^{1 / 2} N\left[-i\left(-n^{2} z^{-1}\right)\right]^{1 / 2} N(\mathfrak{n})^{-1} \vartheta_{\chi}\left(-n^{2} z^{-1}, \mathfrak{n}^{-1}\right), \\
\vartheta_{\bar{\chi}}\left(-n^{2} z^{-1}, \mathfrak{n}^{-1}\right)=\chi(-1) \tau_{n}(\bar{\chi}) D^{1 / 2} N(-i z)^{1 / 2} \vartheta_{\chi}(z, \mathfrak{o})
\end{gathered}
$$

so $\vartheta_{\chi}(z, \mathfrak{o})=\tau_{n}(\chi) \tau_{n}(\bar{\chi}) N((n) \mathfrak{n}) D \vartheta_{\chi}(z, \mathfrak{o})=\left|\tau_{n}(\chi)\right|^{2} N(\mathfrak{m})^{-1} \vartheta_{\chi}(z, \mathfrak{o})$ (we used (11) and (4)). Cancelling the nonzero function $\vartheta_{\chi}(z, o)$, we obtain the desired result. In the case $\chi$ is odd, apply the same argument to the function $\vartheta_{\chi}^{(1)}(z, \mathfrak{o})$ (see next section).

\section{Functions $\vartheta_{\chi}^{(\mu)}\left(z,\left(\begin{array}{l}u \\ v\end{array}\right), \mathfrak{a}\right)$}

In our proof of the main result in $\S 10$, we will encounter the usual difficulty owing to the fact that $\vartheta_{\chi}(z, \mathfrak{a})$ vanishes identically for odd $\chi$. To deal with odd characters, we introduce a new class of theta functions and prove an inversion formula for them, using a well-known trick due to Hecke [3, p. 265].

Fix a number $\mu$ between 1 and $N$, and define

$$
\begin{aligned}
\vartheta_{\chi}^{(\mu)}\left(z,\left(\begin{array}{c}
u \\
v
\end{array}\right), \mathfrak{a}\right) \\
\quad=\left\{\begin{array}{r}
\sum_{a \in \mathfrak{a}} \chi(a)\left(a^{(\mu)}+v^{(\mu)}\right) e_{1}\left[x(a+v)^{2}+i y|a+v|^{2}-2 u a-u v\right], \\
\text { if } \mu \leq r_{1}, \\
\sum_{a \in \mathfrak{a}} \chi(a)\left(\frac{a^{(\mu)}+v^{(\mu)}}{a^{(\mu)}}+\frac{v^{(\mu)}}{2}\right) e_{1}\left[x(a+v)^{2}+i y|a+v|^{2}-2 u a-u v\right], \\
\text { if } \mu>r_{1},
\end{array}\right.
\end{aligned}
$$


where the notation is as in $\S 8$. Note that for $\mu>r_{1}$, the defining expression for $\vartheta_{\chi}^{(\mu)}$ contains a $2 \times 1$ matrix so that $\vartheta_{\chi}^{(\mu)}$ is a $\mathbb{C}^{2}$-valued function!

Again, we will write $\vartheta_{\chi}^{(\mu)}(z, \mathfrak{a})$ if $u=v=0$. Note that $\vartheta_{\chi}^{(\mu)}(z, \mathfrak{a}) \equiv 0$ if and only if $\chi$ is even.

Proposition 5a. Under the same hypotheses as in Proposition 5,

$$
\begin{aligned}
& \vartheta_{\bar{\chi}}^{(\mu)}\left(-n^{2} z^{-1},\left(\begin{array}{c}
-n v \\
0
\end{array}\right), \mathfrak{n}^{-1} \mathfrak{a}^{-1}\right) \\
& =\left\{\begin{array}{rr}
\chi(-1) \tau_{n}(\bar{\chi}) N(\mathfrak{a}) D^{1 / 2} N(-i z)^{1 / 2} \frac{z^{(\mu)}}{n^{(\mu)}} \vartheta_{\chi}^{(\mu)}\left(z,\left(\begin{array}{c}
0 \\
v
\end{array}\right), \mathfrak{a}\right), & \text { if } \mu \leq r_{1}, \\
\chi(-1) \tau_{n}(\bar{\chi}) N(\mathfrak{a}) D^{1 / 2} N(-i z)^{1 / 2} & \\
\times\left(\begin{array}{cc}
n^{(\mu)} x^{(\mu)} & i n^{(\mu)^{-1}} y^{(\mu)} / 2 \\
i n^{(\mu)^{-1}} y^{(\mu)} / 2 & \frac{n^{(\mu)^{-1}}}{\chi^{(\mu)}}
\end{array}\right) \vartheta_{\chi}^{(\mu)}\left(z,\left(\begin{array}{l}
0 \\
v
\end{array}\right), \mathfrak{a}\right), \\
\text { if } \mu>r_{1} .
\end{array}\right.
\end{aligned}
$$

Proof. In the definition of the theta function $\vartheta_{\chi}\left(z,\left(\begin{array}{l}u \\ v\end{array}\right), \mathfrak{a}\right)$, the characteristics $u=\left(u_{1}, \ldots, u_{n}\right), v=\left(v_{1}, \ldots, v_{n}\right)$ can be more generally considered as $2 \mathrm{~N}$ independent complex variables, with the conventions

$$
\begin{aligned}
& \left|a^{(\nu)}+v^{(\nu)}\right|^{2}=\left(a^{(\nu)}+v^{(\nu)}\right)\left(\overline{a^{(\nu)}}+\overline{v^{(\nu)}}\right), \\
& \overline{v^{(\nu)}}= \begin{cases}v^{(\nu)}, & \text { if } \nu \leq r_{1} \\
v^{\left(\nu+r_{2}\right)}, & \text { if } r_{1}<\nu \leq r_{1}+r_{2} \\
v^{\left(\nu-r_{2}\right)}, & \text { if } r_{1}+r_{2}<\nu \leq N .\end{cases}
\end{aligned}
$$

The inversion formula (10) will still be valid, and hence so will (14). Now to obtain (14a), apply the differential operator

$$
\frac{1}{2 \pi i} \frac{\partial}{\partial v^{(\mu)}} \quad\left(\text { if } \mu \leq r_{1}\right) \quad \text { or } \quad \frac{1}{2 \pi i}\left(\begin{array}{l}
\partial / \partial v^{(\mu)} \\
\partial / \partial v^{(\mu)}
\end{array}\right) \quad\left(\text { if } \mu>r_{1}\right)
$$

to both sides of (14) with $u=0$, and then specialize $u, v$ to be in $K$.

\section{Proof of THE REDUCTION Formula}

Theorem 1. Let $\chi$ be a character mod $\mathfrak{m}$ of conductor $\mathfrak{f}, \psi$ the primitive character mod $f$ associated to $\chi, \Psi$ a Hecke character mod $f$ inducing $\psi$. Let $n \in \mathfrak{m}^{-1} \mathfrak{d}^{-1}$ be a parameter, $\mathfrak{n}=\mathfrak{m} \mathfrak{d}(n)$, let $\mathfrak{q}$ be the product of prime ideals of $\mathfrak{o}$ dividing $\mathfrak{m}$ but not $\mathfrak{f}$, and put $\mathfrak{r}=\mathfrak{m} / \mathfrak{f q}$. Then

$$
\tau_{n}(\chi)= \begin{cases}\tau(\psi) \lambda(n) N(\mathfrak{r}) \mu(\mathfrak{q}) \Psi(\mathfrak{q}) \bar{\Psi}(\mathfrak{b}) \mu[(\mathfrak{q}, \mathfrak{b})] \phi[(\mathfrak{q}, \mathfrak{b})], \\ 0, & \text { if } \mathfrak{r} \not \mathfrak{n} .\end{cases}
$$

$(\tau(\psi)=\tau(\Psi)$ is a normed Gaussian sum defined in $\S 5$.)

Proof. First, we deal with the case of even $\chi$. Consider $\vartheta_{\chi}\left(-n^{2} z^{-1}, \mathfrak{o}\right)$, which is not identically zero. Although we cannot apply the inversion formula (14) to it directly ( $\chi$ is not primitive), we can write it as a linear combination of $\vartheta_{\psi}$ 's or $\vartheta$ 's and then apply the respective inversion formulae. This way we 
will obtain the Fourier expansion of the original function in $x$ in two different ways. Comparing the Fourier coefficients, we will obtain (16).

Let $\tau_{n_{0}}(\psi)$ be a proper Gaussian sum, so that $n_{0} \in \mathfrak{f}^{-1} \mathfrak{d}^{-1}$ and the integral ideal $\mathfrak{n}_{0}=\mathfrak{f} \mathfrak{d}\left(n_{0}\right)$ is prime to $\mathfrak{f}$. Also put $\omega=n / n_{0}$. The first way to proceed is via (15) and (14):

$$
\begin{aligned}
\vartheta_{\chi}\left(-n^{2} z^{-1}, \mathfrak{o}\right)= & \sum_{\mathfrak{b} \mid \mathfrak{q}} \mu(\mathfrak{b}) \vartheta_{\psi}\left(-n^{2} z^{-1}, \mathfrak{b}\right) \\
= & \sum_{\mathfrak{b} \mid \mathfrak{q}} \mu(\mathfrak{b}) \psi(-1) \tau_{n_{0}}(\psi) N\left(\mathfrak{n}_{0}^{-1} \mathfrak{b}^{-1}\right) \\
& \times D^{1 / 2} N\left(-i \omega^{-2} z\right)^{1 / 2} \vartheta_{\bar{\psi}}\left(\omega^{-2} z, \mathfrak{n}_{0}^{-1} \mathfrak{b}^{-1}\right) .
\end{aligned}
$$

Making a change of variables $\mathfrak{c}=\mathfrak{b}^{-1} \mathfrak{q}, b=\omega^{-1} a$, we get

$$
\begin{aligned}
\vartheta_{\chi}\left(-n^{2} z^{-1}, \mathfrak{o}\right)= & \left.\tau_{n_{0}}(\psi) N(\mathfrak{r} \mathfrak{q n})^{-1}\right) D^{1 / 2} N(-i z)^{1 / 2} \\
& \times \sum_{\mathfrak{c} \mid \mathfrak{q}} \mu(\mathfrak{q}) \mu(\mathfrak{c}) N(\mathfrak{q})^{-1} N(\mathfrak{c}) \sum_{b \in \mathfrak{r n}-1} \bar{\psi}(\omega b) e_{1}\left(x b^{2}+i y|b|^{2}\right) \\
= & \tau_{n_{0}}(\psi) N\left(\mathfrak{r n}^{-1}\right) D^{1 / 2} N(-i z)^{1 / 2} \mu(\mathfrak{q}) \\
& \times \sum_{b \in \mathfrak{n} \mathfrak{n}^{-1}} \bar{\psi}(\omega b)\left[\sum_{\mathfrak{c} \mid\left((b) \mathfrak{n} \mathfrak{r}^{-1}, \mathfrak{q}\right)} \mu(\mathfrak{c}) N(\mathfrak{c})\right] e_{1}\left(x b^{2}+i y|b|^{2}\right) .
\end{aligned}
$$

The sum in brackets is easily seen to be equal to $\mu\left[\left((b) \mathfrak{n} \mathfrak{r}^{-1}, \mathfrak{q}\right)\right] \phi\left[\left((b) \mathfrak{n} \mathfrak{r}^{-1}, \mathfrak{q}\right)\right]$. Therefore

$$
\vartheta_{\chi}\left(-n^{2} z^{-1}, \mathfrak{o}\right)=\sum_{a \in \mathfrak{n}^{-1}} c(a) e_{1}\left(x a^{2}+i y|a|^{2}\right)
$$

where

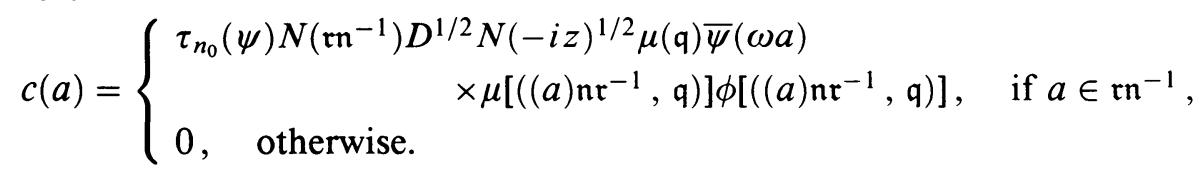

On the other hand, using (13), (12), and (10), we get

$$
\begin{aligned}
& \vartheta_{\chi}\left(-n^{2} z^{-1}, \mathfrak{o}\right)=\sum_{\substack{\mathfrak{b} \in \mathfrak{o} \\
\bmod \mathfrak{m}}} \chi(b) \vartheta\left(-n^{2} z^{-1},\left(\begin{array}{l}
0 \\
b
\end{array}\right), \mathfrak{m}\right) \\
& =\sum_{\substack{b \in \mathfrak{o} \\
\bmod \mathfrak{m}}} \chi(b) \vartheta\left(-z^{-1},\left(\begin{array}{c}
0 \\
n b
\end{array}\right), \mathfrak{n d}^{-1}\right) \\
& =\sum_{\substack{b \in \mathfrak{o} \\
\bmod \mathfrak{m}}} \chi(b) N\left(\mathfrak{n}^{-1}\right) D^{1 / 2} N(-i z)^{1 / 2} \vartheta\left(z,\left(\begin{array}{c}
n b \\
0
\end{array}\right), \mathfrak{n}^{-1}\right) \\
& =N(\mathfrak{n})^{-1} D^{1 / 2} N(-i z)^{1 / 2} \sum_{\substack{b \in \mathfrak{o} \\
\bmod \mathfrak{m}}} \chi(b) \sum_{a \in \mathfrak{n}^{-1}} e_{1}\left(x a^{2}+i y|a|^{2}-2 n b a\right) \\
& =N(\mathfrak{n})^{-1} D^{1 / 2} N(-i z)^{1 / 2} \sum_{a \in \mathfrak{n}^{-1}}\left[\sum_{\substack{b \in \mathfrak{m} \\
\bmod \mathfrak{m}}} \chi(b) e(-n a b)\right] e_{1}\left(x a^{2}+i y|a|^{2}\right) .
\end{aligned}
$$


In other words,

$$
\vartheta_{\chi}\left(-n^{2} z^{-1}, \mathfrak{o}\right)=\sum_{a \in \mathfrak{n}^{-1}} d(a) e_{1}\left(x a^{2}+i y|a|^{2}\right)
$$

where

$$
d(a)=N(\mathfrak{n})^{-1} D^{1 / 2} N(-i z)^{1 / 2} \tau_{-n a}(\chi) .
$$

Comparing the Fourier coefficients at $e_{1}\left(x a^{2}\right)$ in (17) and (18), we get

$$
[c(a)+c(-a)] e_{1}\left(i y a^{2}\right)=[d(a)+d(-a)] e_{1}\left(i y a^{2}\right) .
$$

The assumption that $\chi$ is even implies that $c(-a)=c(a), d(-a)=d(a)$, so $c(a)=d(-a)$. Actually, all we need is the equality $c(1)=d(-1)$. Cancelling common factors, we obtain a formula that expresses $\tau_{n}(\chi)$ in terms of $\tau_{n_{0}}(\psi)$ :

$$
\tau_{n}(\chi)= \begin{cases}\tau_{n_{0}}(\psi) N(\mathfrak{r}) \mu(\mathfrak{q}) \bar{\psi}(\omega) \mu\left[\left(\mathfrak{n} \mathfrak{r}^{-1}, \mathfrak{q}\right)\right] \phi\left[\left(\mathfrak{n} \mathfrak{r}^{-1}, \mathfrak{q}\right)\right], & \text { if } \mathfrak{r} \mid \mathfrak{n} \\ 0, & \text { otherwise. }\end{cases}
$$

Finally, we can get rid of the extra parameter $n_{0}$ and make the formula more transparent by substituting $\tau_{n_{0}}(\psi)=\tau(\psi) \bar{\Psi}\left(\mathfrak{n}_{0}\right) \lambda\left(n_{0}\right)$ (formula (9)) (this, of course, comes at the cost of having to make an arbitrary choice of $\Psi)$. A short calculation shows that if $\mathfrak{n}=\mathfrak{r b}, \mathfrak{b}$ an integral ideal, then

$$
\tau_{n_{0}}(\psi) \bar{\psi}(\omega)=\tau(\psi) \lambda(n) \Psi(\mathfrak{q}) \bar{\Psi}(\mathfrak{b}),
$$

which finishes the proof of (16) in the case $\chi$ is even. If $\chi$ is odd, apply the same argument to the function $\vartheta_{\chi}^{(\mu)}\left(-n^{2} z^{-1}, \mathfrak{o}\right)$ for some $\mu$ (say $\mu=1$ ). (If $\mu>r_{1}$, this is a $\mathbb{C}^{2}$-valued function, so we obtain expansions similar to (17) and (18) and then equate the first coordinates.) The extra factors in the inversion formulas will cancel at the end (we leave the details to the reader). The proof is now complete.

Formula (16) can be stated in a different form: if, with the above notation, we put

then

$$
\mathfrak{m}^{\prime}=\frac{\mathfrak{m}}{(\mathfrak{n}, \mathfrak{m})}, \quad \mathfrak{n}^{\prime}=\frac{\mathfrak{n}}{(\mathfrak{n}, \mathfrak{m})}
$$

$$
\tau_{n}(\chi)= \begin{cases}\tau(\psi) \lambda(n) \frac{\phi(\mathfrak{m})}{\phi\left(\mathfrak{m}^{\prime}\right)} \mu\left(\frac{\mathfrak{m}^{\prime}}{\mathfrak{f}}\right) \Psi\left(\frac{\mathfrak{m}^{\prime}}{\mathfrak{f}}\right) \bar{\Psi}\left(\mathfrak{n}^{\prime}\right), & \text { if } \mathfrak{f} \mid \mathfrak{m}^{\prime}, \\ 0, & \text { otherwise. }\end{cases}
$$

The equivalence of (19) and (16) is proved in [5] for $K=\mathbb{Q}$; the proof for the general case is completely analogous.

Corollary 1. With the above notation, the Gaussian sum $\tau_{n}(\chi)$ does not vanish if and only if $\mathfrak{r} \mid \mathfrak{n}$ and $\left(\mathfrak{n r}^{-1}, \mathfrak{f}\right)=\mathfrak{o}$ (equivalently, if and only if $\mathfrak{f} \mid \mathfrak{m}^{\prime}, \mathfrak{m}^{\prime} / \mathfrak{f}$ is squarefree and $\left.\left(\mathfrak{m}^{\prime} / \mathfrak{f}, \mathfrak{f}\right)=\left(\mathfrak{n}^{\prime}, \mathfrak{f}\right)=\mathfrak{o}\right)$. The absolute value of a nonzero Gaussian sum $\tau_{n}(\chi)$ is equal to $N(\mathfrak{f})^{1 / 2} N(\mathfrak{r}) \phi(\mathfrak{q}, \mathfrak{b})$ (or to $\left.N(\mathfrak{f})^{1 / 2} \phi(\mathfrak{m}) / \phi\left(\mathfrak{m}^{\prime}\right)\right)$.

Proof. Immediate from (16) and (19), together with (7).

With almost no extra effort, Theorem 1 can be generalized to Gaussian sums with Hecke characters, since these can be reduced to Gaussian sums for numerical characters by formula (8).

Corollary 2. Let $X$ be a Hecke character mod $m$ of conductor $\mathfrak{f}$, with infnite part $\lambda, \Psi$ the primitive Hecke character associated to $X$, a a fractional 
ideal, $n \in \mathfrak{m}^{-1} \mathfrak{a}^{-1} \mathfrak{d}^{-1}, \mathfrak{n}=\mathfrak{m a d}(n)$, and let $\mathfrak{q}, \mathfrak{r}$ be defined as in Theorem 1 . Then (16) and (19) still hold with $\tau_{n}(\chi), \tau(\psi)$ replaced by $\tau_{n}(X \mid \mathfrak{a}), \tau(\Psi)$, respectively.

Proof. As we remarked earlier, (19) is equivalent to (16), so we will concentrate on (16). Select an integral ideal $\mathfrak{c}$ such that $(\mathfrak{c}, \mathfrak{m})=\mathfrak{o}$ and $\mathfrak{a} c=(c)$ is principal. By $(8)$,

$$
\tau_{n}(X \mid \mathfrak{a})=X(\mathfrak{c}) \bar{\lambda}(c) \tau_{n c}(\chi)=\Psi(\mathfrak{c}) \bar{\lambda}(c) \tau_{n c}(\chi),
$$

where $\chi$ is the numerical character mod $m$ induced by $X$. (Recall that $X=\Psi$ on $I(\mathfrak{m})$.) Let $\psi$ denote the primitive character associated to $\chi$. Propositions 2 and 3 imply that the infinite part of $\Psi$ equals $\lambda$, the conductor of $\psi$ is $f$, and $\psi$ is the numerical character induced by $\Psi$. Applying Theorem 1 to $\tau_{n c}(\chi)$ and taking into account the formula above, we get

$$
\tau_{n}(X \mid \mathfrak{a})=\left\{\begin{array}{rr}
\tau(\Psi) \lambda(n) N(\mathfrak{r}) \mu(\mathfrak{q}) \Psi(\mathfrak{q}) \overline{\Psi\left(\mathfrak{b}^{\prime} \mathfrak{c}^{-1}\right)} \mu\left[\left(\mathfrak{q}, \mathfrak{b}^{\prime}\right)\right] \phi\left[\left(\mathfrak{q}, \mathfrak{b}^{\prime}\right)\right], \\
0, & \text { if } \mathfrak{r} \backslash \mathfrak{n} \mathfrak{c} .
\end{array}\right.
$$

Since $(\mathfrak{c}, \mathfrak{r})=\mathfrak{o}, \mathfrak{r} \mid \mathfrak{n} \mathfrak{c}$ if and only if $\mathfrak{r} \mid \mathfrak{n}$. Furthermore, if indeed $\mathfrak{n} \mathfrak{c}=\mathfrak{r} \mathfrak{b}^{\prime}$, where $\mathfrak{b}^{\prime}$ is an integral ideal, then the ideal $\mathfrak{b}=\mathfrak{b}^{\prime} \mathfrak{c}^{-1}$ is integral, $\mathfrak{n}=\mathfrak{r b}$ and $\left(\mathfrak{q}, \mathfrak{b}^{\prime}\right)=(\mathfrak{q}, \mathfrak{b})$, so $\tau_{n}(X \mid \mathfrak{a})$ is given by the desired formula.

Putting $\lambda=1$ in the above corollary, we recover Hasse's formula for Gaussian sums with ray class characters $([2$, p. 25], with $v=1)$.

\section{REFERENCES}

1. H. Hasse, Vorlezungen über Zahlentheorie, zweite auf., Springer-Verlag, Berlin, 1964.

2. ___ Allgemeine Theorie der Gaussche Summen in algebraischen Zahlkörpern, Mathematische Abhandlungen, Vol. 3, De Gruyter, Berlin and New York, 1975, pp. 15-39.

3. E. Hecke, Eine neue Art von Zetafunctionen und ihre Beziehungen zur Verteilung der Primzahlen. II, Math. Z. 6 (1920), 11-51.

4. __ Vorlezungen über die Theorie der algebraischen Zahlen, Akademische Verlag, 1923; English transl., Lectures on the theory of algebraic numbers, Springer-Verlag, 1981.

5. H. Joris, On the evaluation of Gaussian sums for non-primitive Dirichlet characters, Enseign. Math. 23 (1977), 13-18.

6. E. Lamprecht, Allgemeine Theorie der Gausssche Summen in endlichen kommutativen Ringen, Math. Nachr. 9 (1953), 149-196.

7. D. Mumford, Tata lectures on theta. I, Birkhäuser, Boston, Mass., 1983.

8. W. Narkiewicz, Elementary and analytic theory of algebraic numbers, PWN, Warsaw, 1974.

9. R. Odoni, On Gauss sums $\left(\bmod p^{n}\right), n \geq 2$, Bull. London Math. Soc. 5 (1973), 325-327.

10. H. Stark, Modular forms and related objects, Number Theory (Montreal, Que., 1985), CMS Conf. Proc., vol. 7, Amer. Math. Soc., Providence, R.I., 1987, pp. 421-455.

Department of Mathematics, Bates College, Lewiston, Maine 04240

Current address: 90 Beaver Street, Apartment 11, Waltham, Massachusetts 02154 